\title{
A laboratory flow reactor with gas particle separation and on-line MS/MS for product identification in atmospherically important reactions
}

\author{
J. F. Bennett ${ }^{1}$, F. Collin ${ }^{1, *}$, and D. R. Hastie ${ }^{1}$ \\ ${ }^{1}$ Department of Chemistry and Centre for Atmospheric Chemistry, York University, 4700 Keele St., Toronto, \\ Ontario, M3J 1P3, Canada \\ *now at: Université Paris Descartes, CNRS UMR 8601, Paris, France
}

Received: 12 May 2009 - Published in Atmos. Meas. Tech. Discuss.: 3 June 2009

Revised: 20 November 2009 - Accepted: 29 November 2009 - Published: 14 December 2009

\begin{abstract}
A system to study the gas and particle phase products from gas phase hydrocarbon oxidation is described. It consists of a gas phase photochemical flow reactor followed by a diffusion membrane denuder to remove gases from the reacted products, or a filter to remove the particles. Chemical analysis is performed by an atmospheric pressure chemical ionization (APCI) triple quadrupole mass spectrometer. A diffusion membrane denuder is shown to remove trace gases to below detectable limits so the particle phase can be studied. The system was tested by examining the products of the oxidation of $\mathrm{m}$-xylene initiated by HO radicals. Dimethylphenol was observed in both the gas and particle phases although individual isomers could not be identified. Two furanone isomers, 5-methyl-2 $(3 \mathrm{H})$ furanone and 3-methyl-2(5H)furanone were identified in the particulate phase, but the isobaric product 2,5 furandione was not observed. One isomer of dimethyl-nitrophenol was identified in the particle phase but not in the gas phase.
\end{abstract}

\section{Introduction}

The oxidation of volatile organic compounds in the atmosphere is a key chemical process in the production of tropospheric ozone and secondary organic particulate matter. Tropospheric ozone has been shown to be a major component of photochemical smog and is responsible for significant negative human health impacts (Bernstein et al., 2004). Particulate matter is increasingly seen as a major environmental

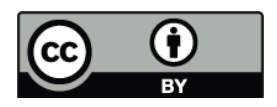

Correspondence to: D. R. Hastie (hastie@yorku.ca) problem, impacting climate, visibility and human health (Dockery et al., 1993; Pöschl, 2005; Krewski et al., 2004).

Secondary particulate matter is that formed by atmospheric gas phase reactions producing low vapour pressure products. Aside from the oxidation of sulfur dioxide and nitrogen oxides, the major contributor to secondary particulate matter is the oxidation of large hydrocarbons. Generally the oxidation of hydrocarbons with less than six carbon atoms does not produce significant amounts of condensable products under atmospheric conditions, but larger hydrocarbons, particularly those that are unsaturated or aromatic can have high yields of condensable products.

To understand and potentially control particulate concentrations in the atmosphere requires a complete understanding of the chemical reactions occurring in their production and the ability to incorporate this knowledge into computer based chemical-meteorological models of the atmosphere. Currently the production of secondary organic particulate matter is not sufficiently well understood to meet this objective. While the rates of oxidation reactions of the parent hydrocarbons are generally well known, (Atkinson and Arey, 2003) the products of these reactions are not nearly as well known. This is particularly true for the larger hydrocarbons as our knowledge of reaction products is mostly extrapolated from the large body of data on small molecule reactions but the larger molecules additionally undergo isomerization and cyclizations that cannot occur in the smaller molecules (Kroll and Seinfeld, 2008). This shortfall in product information has two direct impacts on our ability to construct chemical models. The reaction products undergo further reactions, and inclusion of these reactions requires knowledge of the identity and yields of all such compounds. Even more critical to the ability of the models to predict particle loading is the inclusion of the condensation of these products into the models.

Published by Copernicus Publications on behalf of the European Geosciences Union. 
The strong dependence of vapour pressure on chemical structure means that the identification of products is a critical first step in improving the predictions of particle production algorithms.

Reaction product identification is usually undertaken in laboratory systems by performing reactions under controlled conditions and applying qualitative and quantitative analytical methods to the reaction products. Optical spectroscopic methods, most notably Fourier transform infrared (FTIR), are suitable for simple gas phase molecule identification ( $\mathrm{Li}$ brando and Tringali, 2005; Larsen et al., 2001). Sample collection on filters or cartridges followed by chromatographic separation and mass spectrometric analysis has been the most widely applied method (Larsen et al., 2001; Jaoui and Kamens, 2003; Forstner et al., 1997). Long sampling times mean that large air volumes can easily be sampled and the collected compounds can then be desorbed, either thermally or using solvent, for gas or liquid chromatographic separation. Continuing advances in methods of preparing derivatives of target compounds (Claeys et al., 2007; Temime et al., 2007; Chiappini et al., 2006) and in chromatography, such as 2-D methods (Hamilton et al., 2005), continue to widen the classes of compounds that can be analyzed. Mass spectrometry is now the most common chromatographic detector with MS/MS (Librando and Tringali, 2005) and $\mathrm{MS}^{\mathrm{n}}$ (Larsen et al., 2001) analysis being more widely applied.

On-line mass spectrometry has some significant advantages over other methods. It does not require sample work-up prior to analysis and is not restricted to particular elements or functional groups. Instruments have very fast response times and high sensitivity so that integration times can be kept short allowing reaction products to be followed in real time. The only constraint for on-line mass spectrometry systems is that the sample must be presented to the ion source in the gas phase. This is easily achievable for gas phase products and can be extended to less volatile products by using heated inlets and ion sources. Particulate measurements using a high energy laser for ablation and ionization (Gard et al., 1997; Thomson et al., 2000; Pratt et al., 2009) have been successful although the ionization process results in extensive fragmentation. Two laser systems that separate the ablation and ionization are less destructive, and have been shown to be quantitative for known compounds (Woods et al., 2001). The aerosol mass spectrometer (Drewnick et al., 2005; Allan et al., 2003) has found extensive deployment but the dissociation resulting from the thermal dissociation and electron impact ionization also limits the identification of molecular species. In the gas phase, PTR-MS (Hansel et al., 1995; Tanimoto et al., 2007) utilising low pressure chemical ionization, is potentially valuable especially as the inclusion of high resolution mass spectrometers has allowed single mass peaks to be assigned elemental composition (Jordan et al., 2009). Atmospheric pressure chemical ionization (APCI) methods have been applied sparingly but have shown good results in product identification particularly when coupled to tandem mass spectrometers that can yield structural information on the products. (Hearn and Smith, 2004; Aschmann et al., 2002) Identification and quantitation of reaction products, such as carbonyls, hydroperoxides, and hydroperoxy acids has been demonstrated using an APCI source and the MS/MS capability of an ion trap mass spectrometer (Warscheid et al., 2003; Hoffmann et al., 2002; Reinnig et al., 2009)

Chemical reactors can range from small continuous flow reactors to large batch reactors "smog chambers" that use either natural or simulated light sources. In batch reactors, chemical reactions can proceed from reactants to products under similar conditions to those in the atmosphere, although analytical constraints often require experiments to be done at higher than ambient concentrations. They have large volumes and so there is ample material for analysis making them compatible with the filter and cartridge sampling. They have high volume to surface ratios that lower the importance of complicating wall reactions, and those that rely on sunlight as a light source more closely mimic the real atmosphere. Long sample collection times are needed to collect enough material for most of the analytical methods, and this is usually done by passing the reaction mixture through a collection medium after a reaction has been stopped or has run to completion. This precludes following products through the course of the reaction, i.e. at different reaction times. By contrast, in a continuous flow reactor the reagents enter from one end of the reactor and the reaction proceeds as the reactants flow down the tube. Thus the reaction only proceeds for a time determined by how long the gases spend in the reactor and so they provide complementary strengths to the batch reactor. They can be run for long periods under identical reaction conditions so that large amounts of sample representative of particular reaction conditions can still be collected. By tuning the reaction time samples can be analyzed for intermediate products, which is not usually possible in a batch reactor.

The separation of particles from trace gases for independent analysis of both phases is highly desirable. In particular, the observation of products in the gas phase can be used as a qualitative test of the knowledge of the vapour pressure of a compound and observations in both phases will test our knowledge of equilibrium in the particulate bound system. The particle phase can be easily removed from an air stream using a filter but the reverse problem is more taxing. Since gases have a much higher diffusion coefficient than particles, denuders where the gases preferentially diffuse to absorbing surfaces are the most common method. Surfaces such as charcoal (Temime et al., 2007; Warscheid et al., 2003; Ding et al., 2002) and XAD resin (Temime et al., 2007) can absorb significant amounts of material and have been used extensively. Practical considerations are often a problem where surface absorption can saturate and since the surfaces can be active, gases can be emitted. Rejuvenating these surfaces once contaminated can be difficult. A viable alternative is a diffusion membrane denuder where the wall of the denuder is 


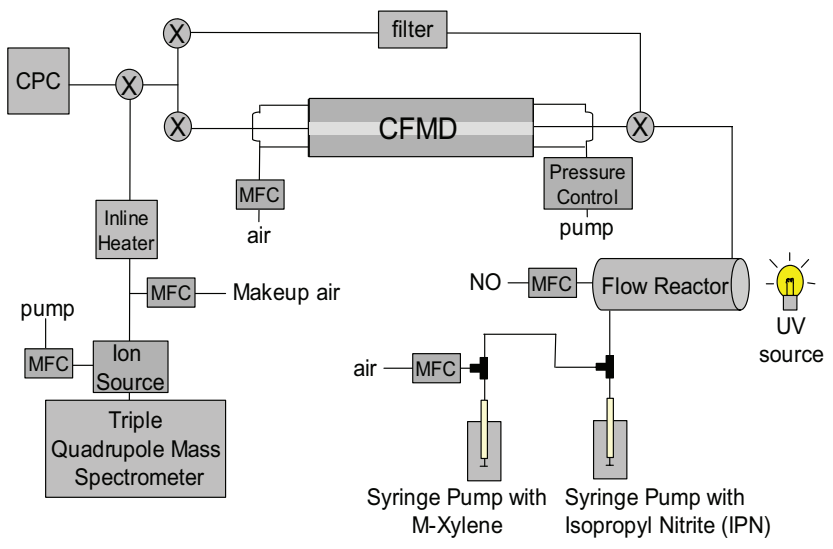

Fig. 1. Experimental set up for photochemical reactions and product identification. (CFMD: the counter flow membrane denuder, MFC: mass flow controller, CPC: condensation particle counter).

a membrane with essentially zero trace gas concentration on the other side so the trace gas diffuses out of the sample flow (Ruiz et al., 2006). Such systems are less likely to become overloaded and so require considerably less maintenance.

In this paper we describe a system to study the products of the reaction of atmospherically relevant hydrocarbons. This system was built to be versatile and incorporates a number of the features described above. It is composed of a gas phase chemical flow reactor which has a UV source to initiate photochemical reactions. This is followed by a diffusion membrane denuder to remove gases from the reacted products, or a filter to remove the particles. Finally the sample is introduced into the atmospheric pressure chemical ionization (APCI) source of a triple quadrupole mass spectrometer for analysis. To demonstrate the value of such a system we present some results from a study of the reaction of $m$-xylene with $\mathrm{HO}$ radicals.

\section{Experimental}

\subsection{System description}

A schematic diagram of the reaction system is shown in Fig. 1. Chemical reactions take place in a photochemical flow reactor. Particles or trace gases are selected for analysis by passing the sample through either a counter flow membrane denuder (CFMD) or a quartz fibre filter respectively. Particle number is measured using a condensation particle counter (CPC) and chemical analysis is performed using a triple quadrupole mass spectrometer.

\subsubsection{Reactor}

The reactor is set up for a continuous flow of air and reagents so the reaction time is constant for a particular experiment. Reactions take place in an $82 \mathrm{~cm}$ long and $6.5 \mathrm{~cm}$ in diameter cylindrical Pyrex reactor with quartz windows at each end. An air flow of $0.60 \mathrm{~L} \mathrm{~min}^{-1}$ is maintained through the reactor by a calibrated flow meter (MKS, Andover MA) to give a residence time of $4.5 \mathrm{~min}$. A comparatively small flow of nitric oxide from a cylinder of $100 \mathrm{ppmv} \mathrm{NO}$ in nitrogen is added to the air flow to produce an NO concentration of $\sim 6 \mathrm{ppmv}$ in the reactor. The hydrocarbon reagent and the isopropyl nitrite (IPN) radical source are added to the air stream at a known rate from individual vials via a syringe pump (Harvard Apparatus Holliston MA) to give nominal concentrations of $100 \mathrm{ppbv}$ and $10 \mathrm{ppmv}$ respectively. The pressure in the cell is maintained at ambient pressure (typically 740 torr) and monitored by a baratron (MKS, Andover MA) pressure gauge.

Light for the photolysis of the IPN (see below) is provided by an Hanovia (1000 W) high pressure xenon arc lamp. This is an ozone free lamp with a UV cut off above $240 \mathrm{~nm}$ so ozone cannot be produced from the photolysis of oxygen. The radiation from the lamp raises the reactor temperature to approximately $26^{\circ} \mathrm{C}$.

The system under study was the oxidation of m-xylene initiated by $\mathrm{HO}$ radicals. The HO radicals were produced photochemically through the photolysis of IPN and subsequent reactions:

$$
\begin{aligned}
& \left(\mathrm{CH}_{3}\right)_{2} \mathrm{CHONO}+h v \rightarrow\left(\mathrm{CH}_{3}\right)_{2} \mathrm{CHO}+\mathrm{NO} \\
& \left(\mathrm{CH}_{3}\right)_{2} \mathrm{CHO}+\mathrm{O}_{2} \rightarrow\left(\mathrm{CH}_{3}\right)_{2} \mathrm{CO}+\mathrm{HO}_{2} \\
& \mathrm{HO}_{2}+\mathrm{NO} \rightarrow \mathrm{HO}+\mathrm{NO}_{2}
\end{aligned}
$$

IPN was chosen as the photochemical HO source because the acetone $\left(\left(\mathrm{CH}_{3}\right)_{2} \mathrm{O}\right)$ produced in Reaction $(\mathrm{R} 2)$ is less reactive than the expected products of the $\mathrm{m}$-xylene oxidation and so should have limited impact on the reaction system. Nitric oxide is added to ensure Reaction (R3) rapidly converts $\mathrm{HO}_{2}$ to $\mathrm{HO}$. The photolysis of $\mathrm{NO}_{2}$ produces ozone through Reactions (R4) and (R5) but the high NO concentration keeps its concentration low, through Reaction (R6):

$$
\begin{aligned}
& \mathrm{NO}_{2}+h v \rightarrow \mathrm{NO}+\mathrm{O} \\
& \mathrm{O}+\mathrm{O}_{2}+M \rightarrow \mathrm{O}_{3}+M \\
& \mathrm{NO}+\mathrm{O}_{3} \rightarrow \mathrm{NO}_{2}+\mathrm{O}_{2}
\end{aligned}
$$

\subsubsection{Separating gas and particles}

Particles can be removed from the air stream by passage through a $25 \mathrm{~mm}$ in-line quartz fibre filter (Pallflex Tissuquartz, Pall, East Hills NY). We assume that the particulate matter on the filter remains in equilibrium with the gas phase species. Since the conditions in the flow reactor do not change within a particular experiment, this is a reasonable assumption. 


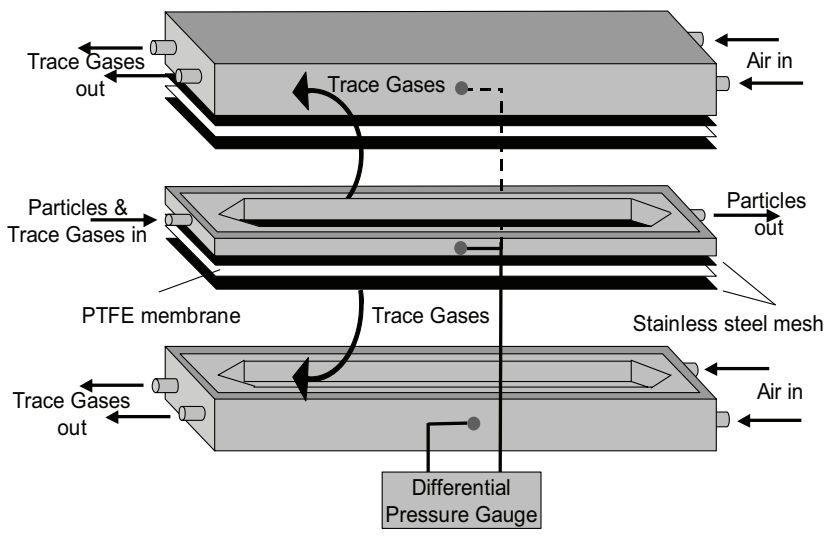

Fig. 2. Exploded diagram of the counter flow membrane denuder. The directions of the air flows are shown as are the desired outcomes of the diffusion.

Trace gases are removed from the air stream using a counter-current flow membrane denuder (CFMD). This follows that described by Ruiz et al. (2006) and a schematic diagram is shown in Fig. 2. The denuder consists of three machined aluminum blocks separated by membranes to give $90 \mathrm{~cm}$ long by $10 \mathrm{~cm}$ wide channels. The centre channel is $1 \mathrm{~cm}$ deep and is for the sample gas, while the two outer channels are $1.5 \mathrm{~cm}$ deep and are for the purified air. The PTFE membrane (Porex PM23J 2 micron pore size, $0.25 \mathrm{~mm}$ thickness, Fairburn GA) is supported on 90 mesh stainless steel for mechanical strength. Access to the sample and air channels are through Swagelok ${ }^{\circledR}$ tube to pipe thread unions. The sample and purified air flow through their respective channels in opposite directions. Inconsistent denuder performance in early experiments was attributed to pressure differences across the membranes. This is now monitored by a differential pressure gauge (Omega PX2650-2BD5V $\pm 500 \mathrm{~Pa}$, Stamford CT) connected between the central and one of the outer channels, so such problems can be readily identified.

The performance of the CFMD is described by the penetration $P_{n}$ which is the ratio of the number of particles exiting the denuder $\left(n_{\text {out }}\right)$ to the number entering $\left(n_{\text {in }}\right)$. This can be estimated assuming a laminar flow in the centre channel and that the transmission of molecules is through a rectangular cross section tube with perfectly adsorbing walls. The concentration at the walls will never be truly zero, however the counter directional flow of the sample from the air ensures that the maximum concentration gradient will be achieved across the membrane at all positions. For the ideal case the penetration $P_{n}$, can be determined from the dimensionless parameter $\mu$ using the equations in Soderholm (1979) as modified in Zhang (2005).

$\mu=\frac{8 D_{p} L W}{3 Q H}$

where $H, W$, and $L$ are the height, width and length of the centre channel respectively, $Q$ is the flow rate through the
Table 1. Determination of the penetration of molecules and particles through the CFMD. Values of $D_{p}$ come from gas phase theory (for the first two entries) and $k T C_{c} / 3 \pi \eta d$ ( $k$ : boltzmann constant, $T$; Temperature, $C_{c}$ : slip correction, $\eta$ air viscosity) for the remainder.

\begin{tabular}{cccc}
\hline $\begin{array}{c}\text { Particle } \\
\text { diameter } \\
d(\mu \mathrm{m})\end{array}$ & $\begin{array}{c}\text { Diffusion coefficient } \\
D_{p}\left(\mathrm{~cm}^{-2} \mathrm{~s}^{-1} @ 298 \mathrm{~K}\right)\end{array}$ & $\mu$ & $\begin{array}{c}\text { Penetration } \\
P_{n}\end{array}$ \\
\hline 0.00037 & $1.9 \mathrm{E}-01$ & $4.6 \mathrm{E}+01$ & $9.1 \mathrm{E}-57$ \\
0.001 & $1.2 \mathrm{E}-02$ & $3.0 \mathrm{E} 00$ & $2.0 \mathrm{E}-04$ \\
0.01 & $5.3 \mathrm{E}-04$ & $1.3 \mathrm{E}-01$ & $6.3 \mathrm{E}-01$ \\
0.015 & $2.4 \mathrm{E}-04$ & $5.8 \mathrm{E}-02$ & $7.8 \mathrm{E}-01$ \\
0.02 & $1.4 \mathrm{E}-04$ & $3.3 \mathrm{E}-02$ & $8.5 \mathrm{E}-01$ \\
0.05 & $2.4 \mathrm{E}-05$ & $5.7 \mathrm{E}-03$ & $9.5 \mathrm{E}-01$ \\
0.1 & $6.8 \mathrm{E}-06$ & $1.6 \mathrm{E}-03$ & $9.8 \mathrm{E}-01$ \\
0.5 & $6.3 \mathrm{E}-07$ & $1.5 \mathrm{E}-04$ & $1.0 \mathrm{E}+00$ \\
1 & $2.8 \mathrm{E}-07$ & $6.6 \mathrm{E}-05$ & $1.0 \mathrm{E}+00$ \\
5 & $4.9 \mathrm{E}-08$ & $1.2 \mathrm{E}-05$ & $1.0 \mathrm{E}+00$ \\
10 & $2.4 \mathrm{E}-08$ & $5.8 \mathrm{E}-06$ & $1.0 \mathrm{E}+00$ \\
50 & $4.8 \mathrm{E}-09$ & $1.1 \mathrm{E}-06$ & $1.0 \mathrm{E}+00$ \\
\hline
\end{tabular}

channel and $D_{p}$ is the diffusion coefficient for the particle in air.

$$
\begin{aligned}
& P_{n}=\frac{n_{\text {out }}}{n_{\text {in }}}=1-1.526 \mu^{\frac{2}{3}}+0.15 \mu+0.0342 \mu^{\frac{4}{3}} \\
& \quad \text { for } \mu<0.05
\end{aligned}
$$

or

$$
\begin{aligned}
P_{n} & =0.9104 \exp (-2.8278 \mu)+0.0531 \exp (-32.147 \mu) \\
& +0.01528 \exp (-93.475 \mu)+0.00681 \exp (-186.805 \mu) \\
& \text { for } \mu>0.05
\end{aligned}
$$

Some representative calculations using the above assumptions are presented in Table 1. The two lowest diameters represent gas molecules of nitrogen and a molecule with a mass 20 times higher, with the remaining entries encompassing representative atmospheric particle sizes. The table shows less than $1 \%$ of trace gases exit the centre channel of the denuder but that more than $80 \%$ of particles of diameter greater than $0.015 \mu \mathrm{m}$ will be transmitted.

The denuder has been designed to effectively remove the trace gases. It could, however, also remove material that evaporates off the particles in the CFMD. Maxwell's equation (Hinds, 1999) can be used to estimate the loss of material. For a pure solvent in which evaporation is controlled by the loss of gas phase molecules by diffusion, the evaporation rate is a function of the droplet diameter $d$, the diffusion coefficient of the evaporating molecule in air $D_{p}$, and the difference between the vapour pressure of the species at the droplet surface $p_{s}$ and the ambient gas pressure $p_{l}$ (assumed to be zero here).

$I=\frac{2 \pi D_{p} M d}{R T}\left(p_{s}-p_{l}\right)$ 
For a $70 \mathrm{~nm}$ particle made up of a compound of molar mass $\mathrm{MM}=100 \mathrm{~g}$ with a saturated vapour pressure of $p_{s}=$ $1 \times 10^{-10} \mathrm{~atm}(0.1 \mathrm{ppbv})$ at $298 \mathrm{~K}$, the loss rate from the particle is approximately $0.12 \% \mathrm{~s}^{-1}$. For such a compound up to $10 \%$ of the original material will be lost to evaporation in this system. Thus this system is not optimized to preserve particle mass, but is perfectly adequate for qualitative product identification.

\subsubsection{In-line heater}

Particles can be heated to drive volatile compounds from the particle phase into the gas phase for analysis by the mass spectrometer. The heater is an $8 \mathrm{~cm}$ long by $0.635 \mathrm{~cm}$ diameter quartz tube surrounded by a heating coil controlled by an in house proportional thermocouple heater controller.

\subsubsection{Triple quadrupole mass spectrometer}

Chemical identification of the reagents and reaction products was undertaken by an APCI triple quadrupole mass spectrometer (TAGA 6000E, MDS Sciex (Dawson et al., 1982). This instrument is configured for atmospheric trace gas analysis and to obtain the required flow through the ion source the sample flow is diluted with purified air. For particle phase measurements the inlet to the instrument was heated to evaporate partially volatile species for analysis. Ionization is achieved by a corona discharge in the sample gas. Since this takes place in air, and the instrument is being used in the positive ion mode, the relevant primary ions produced in the corona discharge are $\mathrm{N}_{2}^{+}$and $\mathrm{O}_{2}^{+}$which in the presence of water vapour produce intermediate ions $\left(\mathrm{H}_{3} \mathrm{O}^{+}\left(\mathrm{H}_{2} \mathrm{O}\right)_{n}\right.$ $n=0,1,2 \ldots)$. For most hydrocarbons the proton affinity (PA) is greater than that of the hydrated hydronium ion so proton transfer to produce an ion of molar mass plus $1 \mathrm{u}(\mathrm{M}+\mathrm{H})^{+}$is energetically favoured. For hydrocarbons with much lower proton affinities, including $\mathrm{m}$-xylene, the true molecular ion $\left(\mathrm{M}^{+}\right)$is regularly observed. The high pressure in the APCI source allows both ion molecule reactions to produce higher $\mathrm{m} / \mathrm{z}$ ions and fragmentation to produce smaller ions. Typically the ion molecule reactions give addition products (often referred to as clusters). In addition to clusters with water, (Good et al., 1970a, b) protonated molecules of general formula $\left(\mathrm{M}_{1} \mathrm{HM}_{2}\right)^{+}$where $\mathrm{M}_{1}$ and $\mathrm{M}_{2}$ may be the same or different species are observed. The most prominent form of this cluster in the current study involves the highly abundant acetone with other products. Fragmentation in the ion source has been shown to interfere with some MS/MS spectra but its impact on the spectra and compound identification is small.

In this work the instrument was used in both the mass spectrum and tandem mass spectrum (MS/MS) modes. In the mass spectrum mode one of the quadrupoles (Q1) scans the mass spectrum of the sample and the other quadrupoles are set to transmit ions resulting in a conventional mass spectrum. This spectrum is used for the preliminary identification of molecular and cluster species. Since the target products and, in some cases, the reagent can generate ions of the same $\mathrm{m} / \mathrm{z}$, the mass spectrum alone cannot be used to identify isobaric (same molar mass) species. Therefore the mass spectrum can only be used to indicate possible molecular and cluster species. True product identification requires the tandem or MS/MS mode to give structural information on the precursor ion and allows more definitive assignment of the peaks in the mass spectrum. In this mode a single precursor ion is selected in the first quadrupole. It is then injected into the second quadrupole with a known and controllable energy. This quadrupole contains argon at comparatively high pressures so that the precursor ion can be fragmented through collisions with the neutral molecules and focused into the third quadrupole for analysis. Examination of these fragment ions gives structural information on the precursor ion and so allows more definitive assignment of the peaks in the mass spectrum. Further refinement is possible by controlling the energy imparted to the ions prior to entering the collision cell, as stronger bonds require higher collision energies to produce characteristic fragments from this bond cleavage.

\subsubsection{Other instrumentation}

Particle numbers were counted using a condensation particle counter (CPC Model 3775, TSI Shoreview MN). For determining size distributions a differential mobility analyzer (DMA, Model 3071 TSI Shoreview MN) was used to size select particles prior to counting.

\subsubsection{Reagents}

M-xylene (Sigma Aldrich, Toronto 99+\%) was used as received. Isopropyl nitrite was prepared in house (Noyes, 1936) and used without distillation. Air was obtained by purifying room air with an AADCO 737-11 operating without a methane catalyst (AADCO, Cleves $\mathrm{OH}$ ). Nitric oxide came from cylinders of 103 ppmv NO in nitrogen (Air Liquide Toronto).

\subsection{Characterization of the system}

\subsubsection{Reactor}

The concentration of m-xylene was followed by the MS/MS signal originating from the $(\mathrm{M}+\mathrm{H})^{+}$ion at $m / z, 107$ fragmenting to the ion at $m / z 92$ (written as $m / z 107 \rightarrow 92$ ). With the configuration described above, the production of $\mathrm{HO}$ radicals and the reaction time of $4.5 \mathrm{~min}$ resulted in the reactive loss of approximately $50 \%$ of the m-xylene.

Particle production was confirmed using the condensation particle counter and typical number concentrations of $10^{5} \mathrm{~cm}^{-3}$ were obtained. This gave a sample rate of $\sim 1 \times 10^{8}$ particles per scan in the mass spectrometer. The particle size distribution as measured by the DMA/CPC system gives a geometric mean and standard deviation of $128 \mathrm{~nm}$ and 

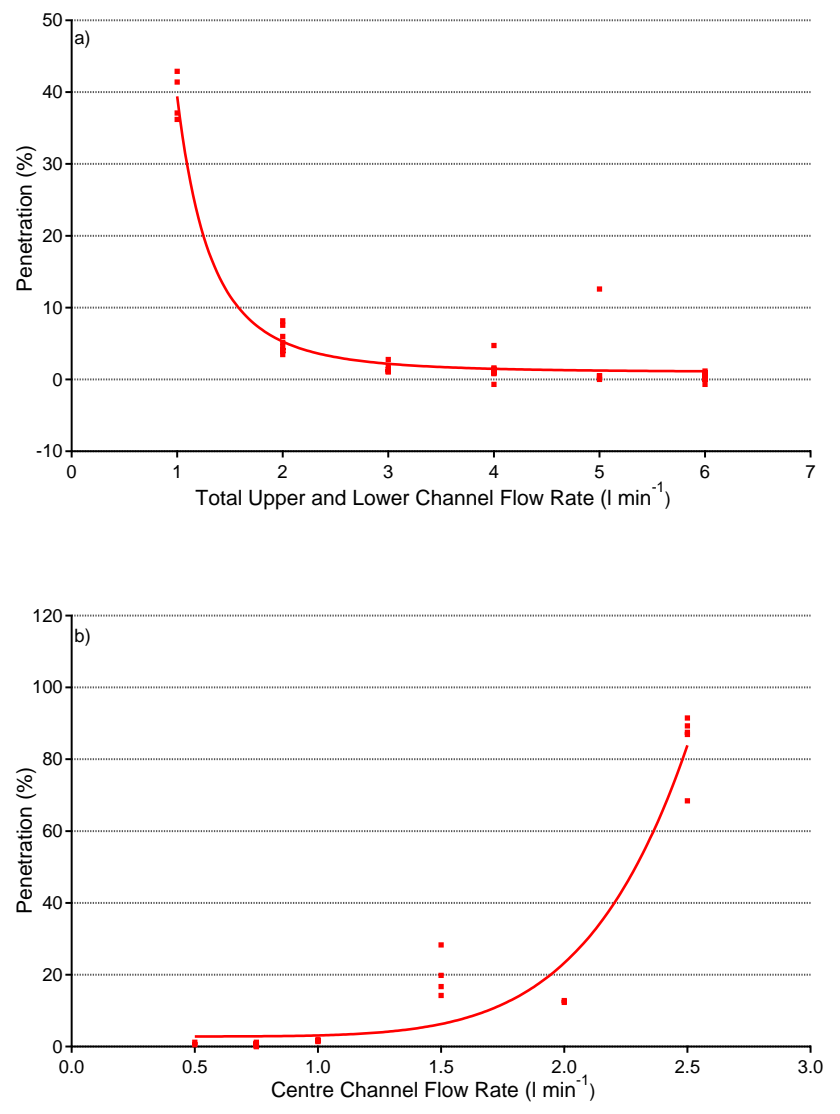

Fig. 3. Penetration of gas phase acetol through the CFMD. (a) Penetration as a function of outer channel flow for a fixed centre channel flow of $0.6 \mathrm{~L} \mathrm{~min}^{-1}$. (b) Penetration as a function of centre channel flow for a fixed outer flow of $6 \mathrm{~L} \mathrm{~min}^{-1}$.

1.66 respectively. The reactor system could be run continuously for several hours, governed by the volume of reagents in the syringe pump.

\subsubsection{CFMD}

Trace gas removal and the mass balance in the CFMD, were determined using gas phase mixtures of 1-hydroxy-2propanone (acetol). This compound was chosen primarily because it was a small multifunctional organic with a useable vapour pressure. Flowing air over a liquid acetol sample and further diluting with air produced an air stream containing approximately $100 \mathrm{ppmv}$ of the acetol. The relative concentrations of the acetol were determined using the TAGA 6000 mass spectrometer in the MS mode by monitoring the protonated acetol at $m / z 75$. Figure 3 shows the penetration of the acetol through the centre channel. In Fig. 3a a fixed flow of $0.6 \mathrm{~L} \mathrm{~min}^{-1}$ was passed through the centre channel, for a residence time of $90 \mathrm{~s}$, and the penetration was determined as a function of the air flow in the outer channels. For flows greater than $2 \mathrm{~L} \mathrm{~min}^{-1}$ there is no measurable acetol left in the centre channel air stream. In Fig. $3 b$ the outer channel
Table 2. Mass balance experiment: \% of acetol recovered in the outer channel flow as a function of that flow.

\begin{tabular}{cc}
\hline $\begin{array}{c}\text { Outer channel flow } \\
\text { L min }\end{array}$ & \% recovery of acetol \\
\hline 2 & $105 \pm 23 \%$ \\
4 & $100 \pm 32 \%$ \\
6 & $88 \pm 25 \%$ \\
\hline
\end{tabular}

flow is maintained at $6 \mathrm{~L} \mathrm{~min}-1$ and the centre channel flow varied. No acetol is detected for centre channel flows less than $1 \mathrm{~L} \mathrm{~min}^{-1}$.

To ensure the trace gas was being removed by diffusion into the outer channel air flow, and not simply adsorbing onto the surfaces of the CFMD, three mass balance experiments were conducted. The concentration of acetol leaving the outer channel of the CFMD was measured and expressed as a percentage of that admitted to the centre channel; the results are presented in Table 2. Admitting the higher flow rates to the dilution system of the TAGA lowers the stability of the measurements so the results are more scattered, however the recovery of the trace gas is within error of $100 \%$. This indicates that adsorption of the trace gas is not a significant loss mechanism, even for a polar oxygenated gas. The conclusion of the gas phase experiments is that for centre channel flows of $\sim 0.6 \mathrm{~L} \mathrm{~min}^{-1}$ and outer channel flows of $6 \mathrm{~L} \mathrm{~min}^{-1}$ the trace gas removal by diffusion gives a sample flow where the trace gas signal is below detectable levels.

These conditions were then tested for the penetration of particles. Ammonium sulfate particles were prepared by passing a flow of $5 \mathrm{~L} \mathrm{~min}^{-1}$ through a $0.5 \mathrm{~g} \mathrm{~L}^{-1}$ ammonium sulfate solution in a Collison nebulizer (May, 1973) (BGI Waltham MA). The resulting droplets were passed through a diffusion dryer containing silica gel which decreased the relative humidity of the aerosol stream to 33-40\%, and through a Po-210 charge neutralizer. The particles were passed through the reactor and the size distribution showed broad polydisperse aerosol with a maximum at $35 \mathrm{~nm}$. Therefore these particles are much smaller than those leaving the reactor and so provide a more stringent test of the CFMD using particles that were inert and did not evaporate. Of this flow, $0.6 \mathrm{~L} \mathrm{~min}^{-1}$ was admitted to the central channel of the CFMD with between 1 and $6 \mathrm{~L} \mathrm{~min}^{-1}$ of purified air in the outer channels. The total number of particles bypassing, or penetrating through the CFMD was measured by the CPC. The penetration of particles through the denuder is shown in Fig. 4 which shows that the particle penetration is indistinguishable from $100 \%$ for our experimental conditions. This denuder has been running continuously for several months without any indication of becoming irreversibly saturated, unlike the XAD coated annular denuders previously in use. 


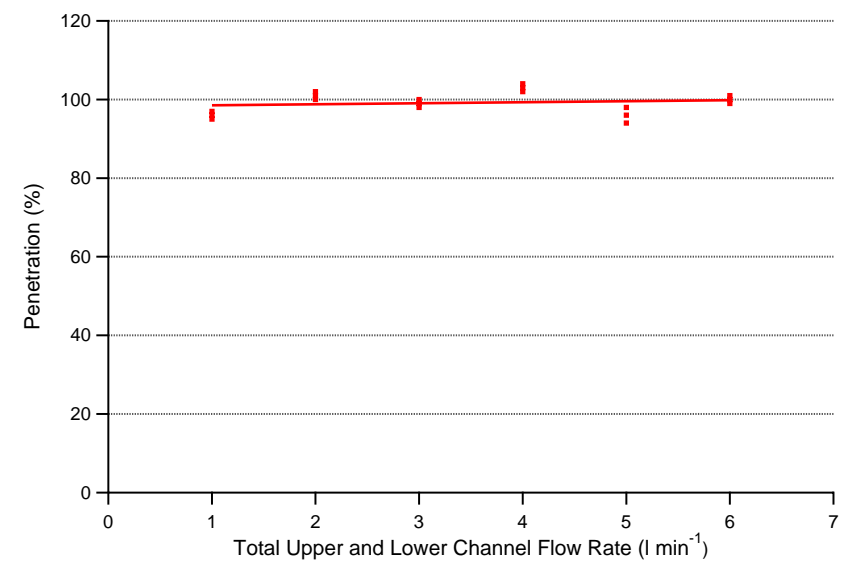

Fig. 4. Penetration of ammonium sulfate particles through the CFMD. The insert shows the size distribution of the particles used for this experiment.

\subsubsection{In-line heater}

The in-line heater was tested on reaction product particles to ensure it could evaporate at least the more volatile material into the ion source. Two particles sizes were sampled by a DMA from those leaving the reactor: 120 and $190 \mathrm{~nm}$. The effect of the heater temperature on the size distribution for the $190 \mathrm{~nm}$ particles is shown in Fig. 5. As the temperature of the heater was increased the diameter and the number of particles decreased, with mean sizes dropping to 50 and $70 \mathrm{~nm}$ by $200^{\circ} \mathrm{C}$. The volume, and therefore mass, decreased markedly by $40 \%$ when the heater was raised by $100^{\circ} \mathrm{C}$ and by $97 \%$ at $200^{\circ} \mathrm{C}$. Thus for these non seeded particles most of the mass is available for analysis provided there is efficient transfer from the heater to the ion source of the mass spectrometer.

\section{Application to the analysis of $m$-xylene oxidation products}

The system has been tested in a study of the oxidation products of the HO initiated oxidation of m-xylene. Within a single experiment, chemical analysis was performed on both trace gas and particle phases. The filtered gas phase products were sampled directly into the TAGA. The particulate phase sample from the CFMD was heated prior to admission to the ion source. Little difference was observed in the observed mass spectra for in-line heater temperatures from 50 to $150^{\circ} \mathrm{C}$, but to minimize concerns about the potential pyrolysis of minor products the results reported here were performed with the inlet heater temperature of $100^{\circ} \mathrm{C}$.

The first step in product identification was to perform an MS mode scan to obtain the mass spectrum of all the ions generated by the APCI source, with true product identification coming from the MS/MS mode. Figure $6 a$ and $b$

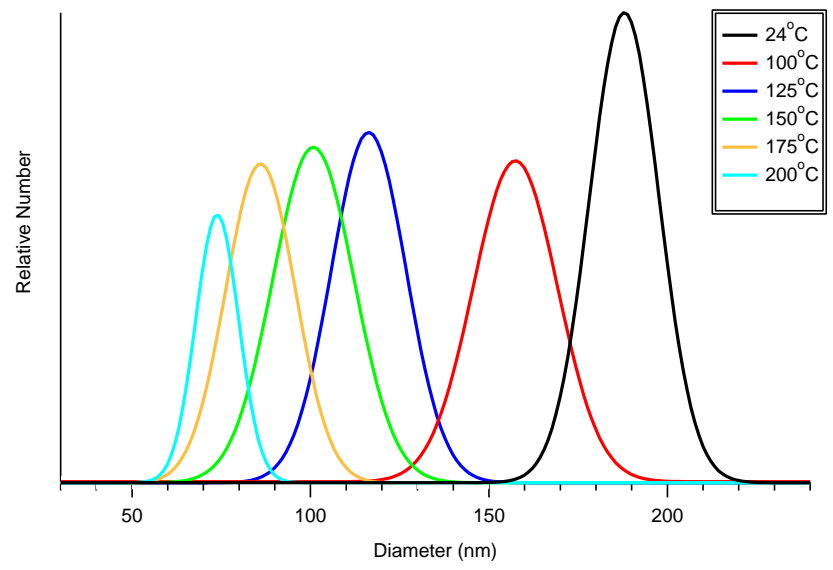

Fig. 5. Effect of in-line temperature on the size distribution of particles leaving the reactor.

shows the MS spectra of the particle phase and gas phase reaction products normalized to the highest signal. Figure $6 \mathrm{c}$ shows the unreacted reagents (in the absence of light) for comparison. The hydrocarbon is barely visible as the $(\mathrm{M}+\mathrm{H})^{+}$ion at $m / z, 107$, consistent with the low proton affinity of aromatic species and the determined detection limit of 10 ppmv. Rather the spectrum is dominated by IPN, acetone and propanol contaminants of the IPN, their dimers and clusters. The product and reagent spectra are vastly different with both gas and particle spectra show significant numbers of ions with $\mathrm{m} / z$ greater than the m-xylene $(\mathrm{M}+\mathrm{H})^{+}$ion at $m / z$ 107. Thus there are multiple ions on which to base product studies.

To test the utility of this system we applied MS/MS to three reaction products, of which each has potentially more than one isomer: dimethylphenol, methyl-furanone, and dimethyl-nitrophenol. At least some of each of these isomers has been previously identified as particulate phase products of smog chamber m-xylene oxidation (Forstner et al., 1997).

The dimethylphenol products result from $\mathrm{HO}$ radical addition to the aromatic ring followed by reaction with $\mathrm{O}_{2}$ or $\mathrm{NO}_{2}$ (Atkinson and Arey, 2003; Andino et al., 1996). Andino et al. suggest the most favourable addition would be ortho to one of the methyl groups with the addition to the 2 position, to produce the 2,6-dimethylphenol slightly more favoured over addition to the 4 position, to produce the 2,4-dimethylphenol. Forstner et al. (1997) identified primarily the 2,4 isomer with a below quantifiable amount of the 2,6 isomer. These products would be expected to be more likely found in the particle phase than the m-xylene as they are more polar and so would have a lower vapour pressure.

The primary ion from dimethylphenol is expected to be the protonated molecule at $m / z 123$. However, the MS/MS spectra showed there are multiple species contributing to $m / z, 123$ in the samples from the reactor. This forced the use of the $\mathrm{M}^{+}$ion at $m / z, 122$ to probe for the presence of 

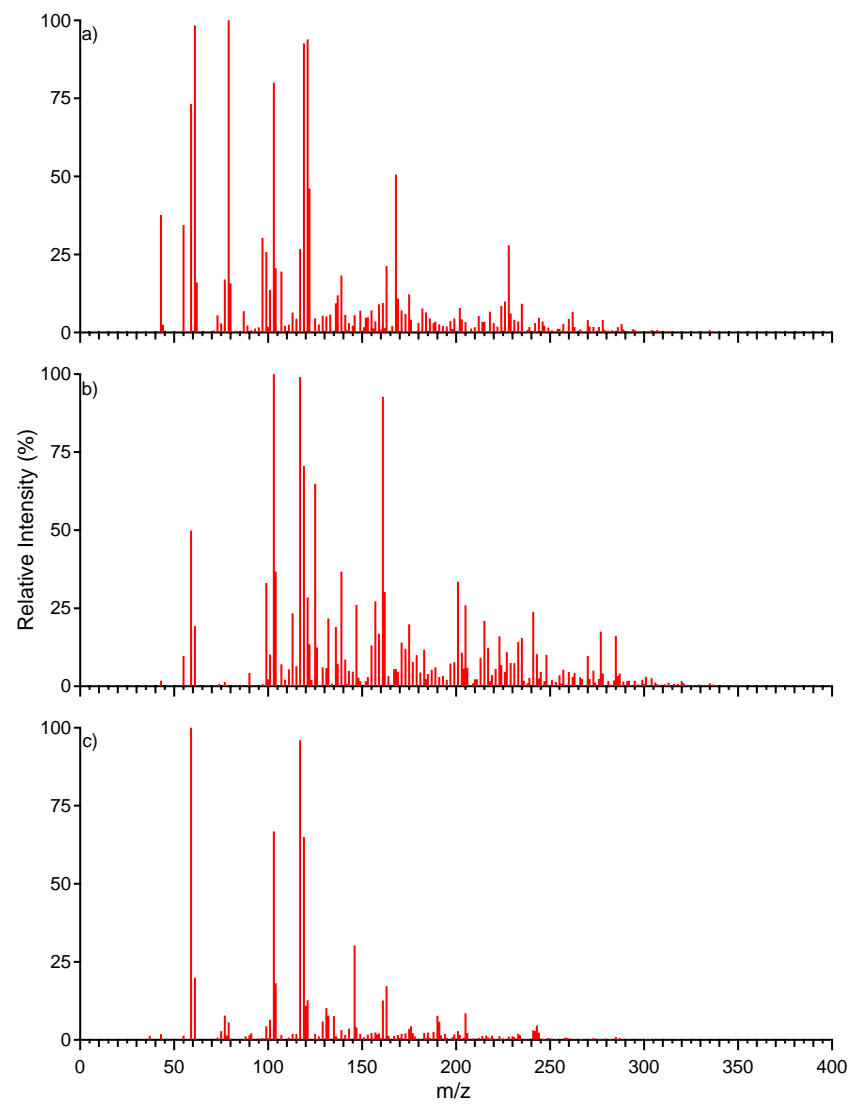

Fig. 6. Mass spectra of samples from the reaction of $m$-xylene with HO radicals (a) particle phase sample (b) the gas phase sample and (c) the unreacted reagents.

dimethylphenol. The MS/MS spectra of $m / z 122$ at three collision energies from the particle phase sample, the gas phase sample, and the headspace gas above authentic samples of 2,6 and 2,4 dimethylphenol are shown in Fig. 7. The MS/MS spectra of the 2,4 and the 2,6 dimethylphenol isomers are not sufficiently different to be useful in distinguishing between the isomers. The major ion peaks in the trace gas spectrum (b), namely $\mathrm{m} / \mathrm{z} 121,107,79$, and 77 , and their variation with collision energy are the same as those in the authentic samples indicating the presence of at least one isomer of dimethylphenol in the gas phase. Based on the same ions, at least one isomer of dimethylphenol is also indicated by the particle phase spectrum. The increased prevalence of the $m / z 43$ ion in the particle phase spectrum remains unexplained. Somewhat surprisingly, removing the heating had little impact on the observed particle phase spectrum. The gas phase product leaving the reactor would have been removed by the CFMD and assuming the particulate phase is not directly vaporized and ionized in the ion source, this signal must originate from gas phase dimethylphenol that evaporates from the particle phase in the CFMD and in the transfer line to the ion source. This is consistent with the

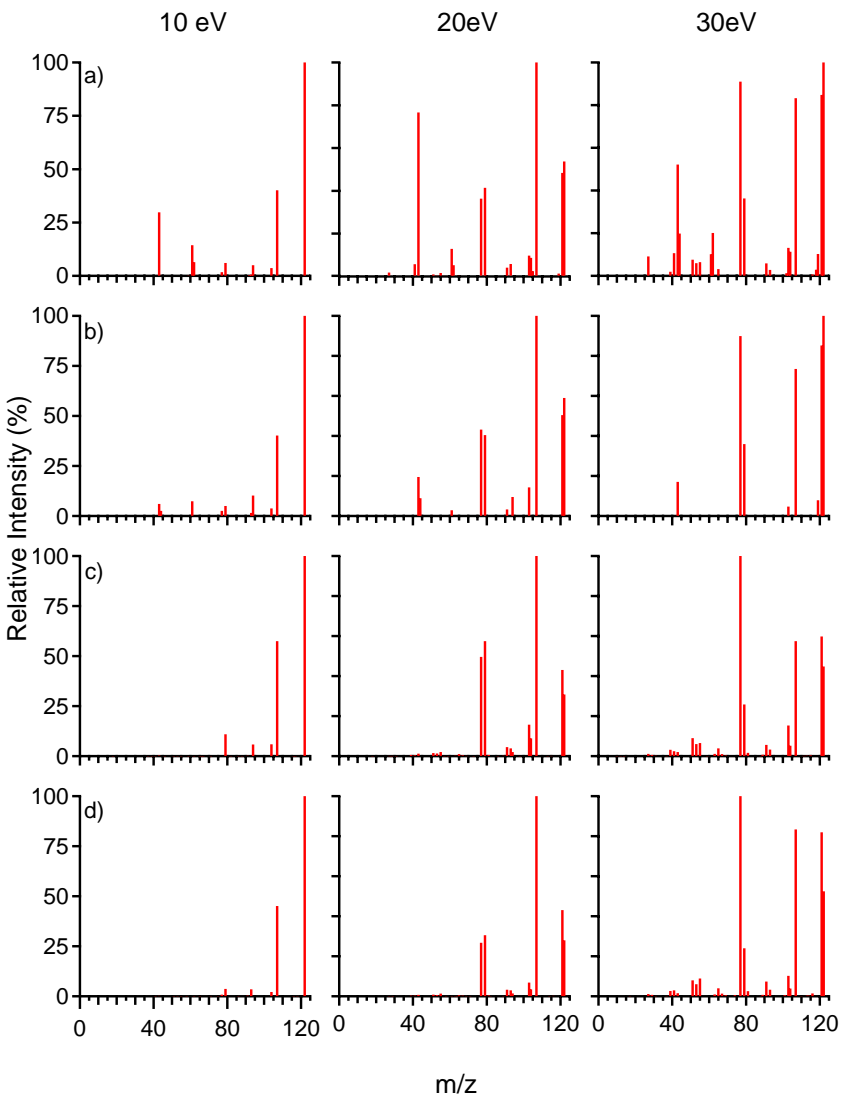

Fig. 7. MS/MS spectra of $m / z$ 122. (a) the particle phase sample, (b) the gas phase sample with no heating prior to analysis, (c) the headspace gas above an authentic sample of 2,6 dimethylphenol. (d) the headspace gas above an authentic sample of 2,4 dimethylphenol.

high vapour pressures of these compounds, $1.7 \times 10^{-4} \mathrm{~atm}$ (170 ppmv) at $25^{\circ} \mathrm{C}$ (CRC handbook). It is also conceivable that these signals could have come directly from particulate phase product entering the ion source. However, this signal still remained despite placing a filter after the CFMD to remove the particulate phase products. This confirms the evaporation of particle phase dimethylphenol occurred in the CFMD, the transfer lines or from filter bound particulate matter.

There are two previously identified methyl-furanones: 5-methyl-2(3H)furanone and 3-methyl-2(5H)furanone (Forstner et al., 1997). These are 5 carbon species resulting from aromatic ring opening followed by a 3 carbon fragment loss and subsequent cyclization. Although of a lower mass than the dimethylphenols these products would also be expected to be present in the particle phase because the multiple functionality would encourage a lower vapour pressure.

The dominant ion from authentic samples of the methylfuranones is the protonated molecule at $\mathrm{m} / \mathrm{z} 99$. Figure 8 shows the MS/MS spectrum of $\mathrm{m} / \mathrm{z}, 99$ from the particle and 


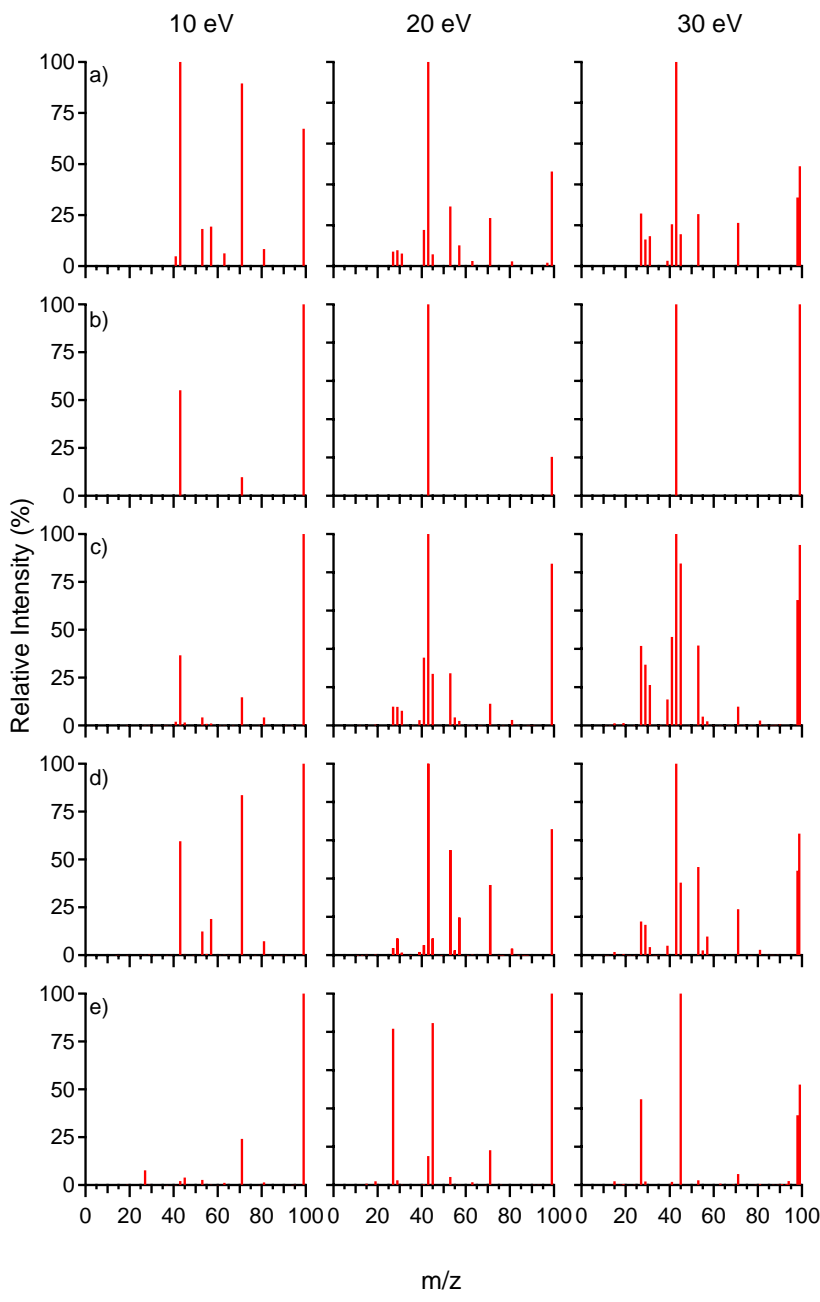

Fig. 8. MS/MS spectra of $m / z 99$ (a) the particle phase sample heated to $100^{\circ} \mathrm{C},(\mathbf{b})$ the gas phase sample (c) an authentic sample of 2,5 furandione (d) 3-methyl-2(5H)furanone and (e) an authentic sample of 5-methyl-2(3H)furanone.

gas phase samples compared with that from the headspace gas above authentic samples of 3-methyl-2(5H)furanone and 5-methyl-2(3H)furanone along with a likely isobaric product, 2,5 furandione identified by Forstner et al. (1997). The authentic samples of the methyl-furanones (Fig. 8c and d) show fragment peaks at $m / z 98,71,53$ and 43 , and the funandione shows fragments at $m / z 71,45$, and 27 . The reaction gas products show only a single fragment ion at $m / z 43$. Therefore there is no evidence for either of the methylfuranones or the furandione in the gas phase reaction products. The MS/MS spectra of the particulate phase products, in Fig. 8a, show the same fragment peaks as both methylfuranone isomers. This is compelling evidence for the presence of at least one of the methyl-furanones. Furthermore there are small differences in the MS/MS spectra of the isomers that allow clarification of the reaction product identity. The peak at $m / z 57$ observed in the sample only appears

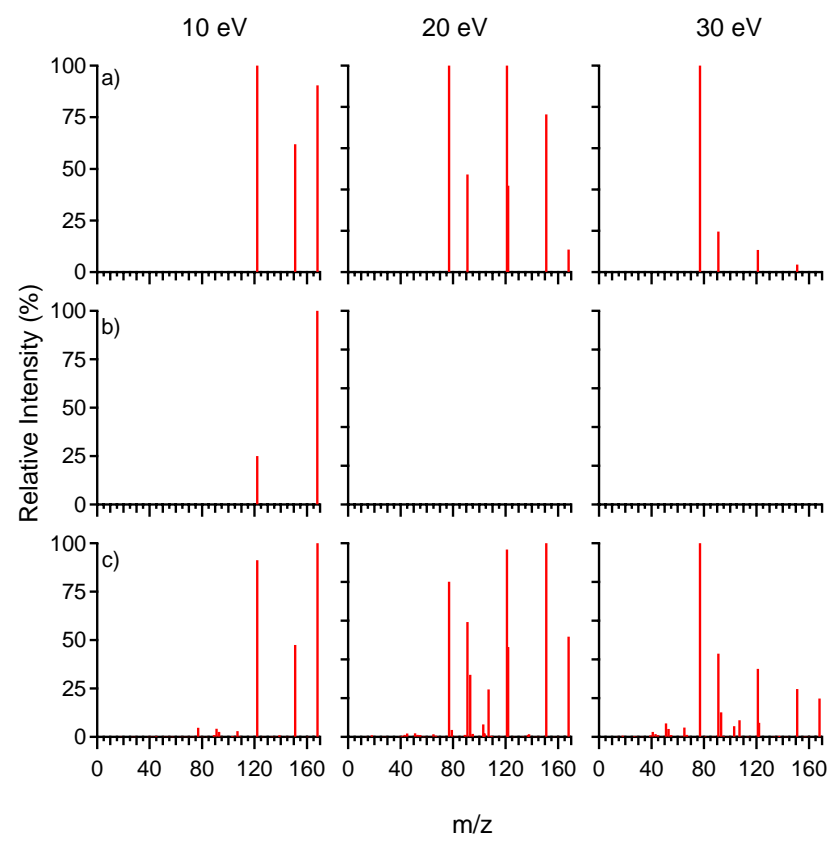

Fig. 9. MS/MS spectra of $m / z 168$ (a) the particle phase sample (b) the gas phase sample (c) an authentic sample of 2,6-dimethyl-4nitrophenol

in the MS/MS spectrum of the 5-methyl-2(3H)furanone and the high relative intensity at $\mathrm{m} / \mathrm{z} 71$ in the spectrum determined at $10 \mathrm{eV}$ of that compound both confirm the presence of this isomer. The peak at $m / z, 41$ only appears in the MS/MS of the 3-methyl-2 $(5 \mathrm{H})$ furanone at collision energies greater than $20 \mathrm{eV}$ confirming its presence. Identifying or eliminating, 2,5 furandione as a possible product requires a unique signature of the furandione. The spectra of the furandione shows a peak at $\mathrm{m} / \mathrm{z}, 27$ that is present at all collision energies with the other major fragment peak at $m / z, 45$ appearing in the $20 \mathrm{eV}$ spectrum and being dominant at $30 \mathrm{eV}$. Each of these $\mathrm{m} / \mathrm{z}$ peaks are very small contributors to the methyl-furanone spectra. In the sample spectra there is no measurable contribution at $m / z 27$ at $10 \mathrm{eV}$ collision energy and $m / z 45$ is a very small contributor at 20 and $30 \mathrm{eV}$. It is never possible to exclude a product with this kind of analysis, it would appear that there is no measurable 2,5 furandione in the sample, but both furanone isomers are present in the particulate phase samples.

The dimethyl-nitrophenols are ring retaining products that can result from the oxidation of one of the dimethylphenol isomers. This necessitates a second radical reaction with the $\mathrm{m}$-xylene reaction product and results in a product that is $60 \mathrm{amu}$ heavier than the parent hydrocarbon and is more polar and so would also be expected to have a lower vapour pressure.

The dominant ion from the only available authentic sample of dimethyl-nitrophenol, 2,6-dimethyl-4-nitrophenol, is the protonated molecule at $m / z 168$. Figure 9 shows the MS/MS 
spectrum of $m / z 168$ from the particulate phase reaction mixture, the gas phase and that from the headspace gas above authentic samples of the 2,6-dimethyl-4-nitrophenol. The $10 \mathrm{eV}$ spectrum of the gas phase sample shows an indication of dimethyl-nitrophenol but generally there was insufficient signal at $\mathrm{m} / z, 168$ to reliably ascribe its presence, as in the 20 and $30 \mathrm{eV}$ spectra shown. The MS/MS spectrum from the particulate phase reaction products shows spectra that have all the major peaks, $m / z 151,122,121,91,76$ over all the collision energies of the authentic sample. Hence it appears that the nitrophenol is only consistently observable in the particulate phase samples. There is no evidence for evaporation of this product in the CFMD or in the transfer lines.

\section{Conclusions}

A laboratory system to study the products of atmospherically important oxidation reactions has been described. Reactions are carried out in a photochemical flow reactor which has the advantage over batch reactors of providing a continuous supply of products for analysis. Changes in flow rate can be used to vary the reaction time but practical considerations mean they must be close to the current value of $4.5 \mathrm{~min}$. A robust membrane denuder that requires almost no maintenance can separate the particle products from the accompanying trace gases without evidence of denuder loading or breakthrough. With the alternative of filtering the particles from the gases, either gas or particle phase products can be selected for analysis. A triple quadrupole mass spectrometer utilizing atmospheric pressure chemical ionization was used primarily in the MS/MS mode for product analysis. For complex mixtures, the low degree of fragmentation in the APCI source, compared to electron impact for example, gives mass spectra of fewer peaks that are more easily interpreted. MS/MS spectra of the sample can be compared with those from authentic samples to assign spectra to specific products. Furthermore the structural information resulting from the MS/MS spectra can be used to infer the identity of products for which no standards are available.

The system has been used to study the HO initiated oxidation of $\mathrm{m}$-xylene. Three possible reaction products were targeted to test the system. These products were identified by comparison of the MS/MS spectra of the reaction products against those from authentic standards. Dimethylphenol is a low volatility product that was identified in both gas and particle phases. The MS/MS spectra of the isomers are not sufficiently different to allow them to be separately identified. Qualitative information on the high volatility of the dimethylphenol was inferred from its continued gas phase presence after the trace gases were removed by the denuder. Two isomeric methyl-furanones have been identified in the particle phase but are not present in the gas phase. Attempts to identify the isobaric furandione were not successful in either gas or particle phase implying that, if present, it is a minor product. Dimethylnitrophenol that is probably formed from further reaction of dimethylphenol was also only observed in the particle phase.

The performance of this combination of reactor, denuder and mass spectrometer has demonstrated it can contribute to the identification of gas and particle phase products of atmospherically important reactions

Acknowledgements. This work was done with support from the National Sciences and Engineering Research Council of Canada (NSERC), the Canadian Foundation for Climate and Atmospheric Sciences (CFCAS), and the Ontario Ministry of the Environment. Contributions from J. Auld, N. Karellas, and J. Merritt are gratefully acknowledged.

Edited by: H. Schlager

\section{References}

Allan, J. D., Jimenez, J. L., Williams, P. I., Alfarra, M. R., Bower, K. N., Jayne, J. T., Coe, H., and Worsnop, D. R.: Quantitative sampling using an Aerodyne aerosol mass spectrometer: 1. techniques of data interpretation and error analysis, J. Geophys. Res. Atmos., 108, 4090, doi:10.1029/2003JD001607, 2003.

Andino, J. M., Smith, J. N., Flagan, R. C., Goddard III, W. A., and Seinfeld, J. H.: Mechanism of atmospheric photooxidation of aromatics: A theoretical study, J. Phys. Chem., 100, 1096710980, 1996.

Aschmann, S. M., Atkinson, R., and Arey, J.: Products of reaction of $\mathrm{OH}$ radicals with alpha-pinene, J. Geophys. Res. Atmos., 107, 4191, doi:10.1029/2001JD001098, 2002.

Atkinson, R. and Arey, J.: Atmospheric degradation of volatile organic compounds, Chem. Rev., 103, 4605-4638, 2003.

Bernstein, J. A., Alexis, N., Barnes, C., Bernstein, I. L., Bernstein, J. A., Nel, A., Peden, D., Diaz-Sanchez, D., Tarlo, S. M., and Williams, P. B.: Health effects of air pollution, J. Allergy Clin. Immun., 114, 1116-1123, 2004.

Chiappini, L., Perraudin, E., Durand-Jolibois, R., and Doussin, J. F.: Development of a supercritical fluid extraction-gas chromatography-mass spectrometry method for the identification of highly polar compounds in secondary organic aerosols formed from biogenic hydrocarbons in smog chamber experiments, Anal. Bioanal. Chem., 386, 1749-1759, 2006.

Claeys, M., Szmigielski, R., Kourtchev, I., Van der Veken, P., Vermeylen, R., Maenhaut, W., Jaoui, M., Kleindienst, T. E., Lewandowski, M., Offenberg, J. H., and Edney, E. O.: Hydroxydicarboxylic acids: Markers for secondary organic aerosol from the photooxidation of $\alpha$-pinene, Environ. Sci. Technol., 41, 1628-1634, 2007.

Dawson, P. H., French, J. B., Buckley, J. A., Douglas, D. J., and Simmons, D.: The use of triple quadrupoles for sequential mass spectrometry: 2-A detailed case study, Org. Mass Spectrom., 17, 212-219, 1982.

Ding, Y. M., Pang, Y. B., Eatough, D. J., Eatough, N. L., and Tanner, R. L.: High-volume diffusion denuder sampler for the routine monitoring of fine particulate matter: II. field evaluation of the PC-BOSS, Aerosol Sci. Tech., 36, 383-396, 2002. 
Dockery, D., Pope, C. A., Xu, X., Spengler, J., Ware, J., Fay, M., Ferris, B., and Speizer, F.: An association between air pollution and mortality in six US cities, N. Engl. J. Med., 329, 1753-1759, 1993.

Drewnick, F., Hings, S. S., DeCarlo, P., Jayne, J. T., Gonin, M., Fuhrer, K., Weimer, S., Jimenez, J. L., Demerjian, K. L., Borrmann, S., and Worsnop, D. R.: A new time-of-flight aerosol mass spectrometer (TOF-AMS) - instrument description and first field deployment, Aerosol Sci. Tech., 39, 637-658, 2005.

Forstner, H. J. L., Flagan, R. C., and Seinfeld, J. H.: Secondary organic aerosol from the photooxidation of aromatic hydrocarbons, Molecular composition, Env. Sci. Tech., 31, 1345-1358, 1997.

Gard, E., Mayer, J. E., Morrical, B. D., Dienes, T., Fergenson, D. P., and Prather, K. A.: Real-time analysis of individual atmospheric aerosol particles: Design and performance of a portable ATOFMS, Anal. Chem., 69, 4083-4091, 1997.

Good, A., Durden, D. A., and Kebarle, P.: Mechanism and rate constants of ion-molecule reactions leading to formation of $\mathrm{H}^{+}\left(\mathrm{H}_{2} \mathrm{O}\right)_{n}$ in moist oxygen and air, J. Chem. Phys., 52, 222 229, 1970a.

Good, A., Durden, D. A., and Kebarle, P.: Ion-molecule reactions in pure nitrogen and nitrogen containing traces of water at total pressures $0.5-4$ torr. kinetics of clustering reactions forming $\mathrm{H}^{+}\left(\mathrm{H}_{2} \mathrm{O}\right)_{n}$, J. Chem. Phys., 52, 212-221, 1970 b.

Hamilton, J., Webb, P., Lewis, A., and Reviejo, M.: Quantifying small molecules in secondary organic aerosol formed during the photo-oxidation of toluene with hydroxyl radicals, Atmos. Environ., 39, 7263-7275, 2005.

Hansel, A., Jordan, A., Holzinger, R., Prazeller, P., Vogel, W., and Lindinger, W.: Proton transfer reaction mass spectrometry: Online trace gas analysis at the ppb level, Int. J. Mass Spectrom. Ion Processes, 149/150, 609-619, 1995.

Hearn, J. D. and Smith, G. D.: A chemical ionization mass spectrometry method for the online analysis of organic aerosols, Anal. Chem., 76, 2820-2826, 2004.

Hinds, W. C.: Aerosol Technology: Properties, Behavior, and Measurement of Airborne Particles, 2nd Edn., Wiley, New York, 1999.

Hoffmann, T., Bandur, R., Hoffmann, S., and Warscheid, B.: Online characterization of gaseous and particulate organic analytes using atmospheric pressure chemical ionization mass spectrometry, Spectrochim. Acta, Part B, 57B, 1635-1647, 2002.

Jaoui, M. and Kamens, R. M.: Mass balance of gaseous and particulate products from $\beta$-Pinene $/ \mathrm{O}_{3} /$ Air in the absence of light and $\beta$-Pinene/NOx/Air in the presence of natural sunlight, J. Atmos. Chem., 45, 101-141, 2003.

Jordan, A., Haidacher, S., Hanel, G., Hartungen, E., Märk, L., Seehauser, H., Schottkowsky, R., Sulzer, P., and Märk, T. D.: A high resolution and high sensitivity proton-transfer-reaction time-of-flight mass spectrometer (PTR-TOF-MS), Int. J. Mass Spectrom., 286, 122-128, 2009.

Krewski, D., Burnett, R. T., Goldberg, M. S., Hoover, K., Siemiatycki, J., Abrahamowicz, M., and White, W. H.: Validation of the Harvard six cities study of particulate air pollution and mortality, N. Engl. J. Med., 350, 198-199, 2004.
Kroll, J. H. and Seinfeld, J. H.: Chemistry of secondary organic aerosol: Formation and evolution of low-volatility organics in the atmosphere, Atmos. Environ., 42, 3593-3624, 2008.

Larsen, B. R., DiBella, D., Glasius, M., Winterhalter, R., Jensen, N. R., and Hjorth, J.: Gas-phase $\mathrm{OH}$ oxidation of monoterpenes: Gaseous and particulate products, J. Atmos. Chem., 38, 231-276, 2001.

Librando, V. and Tringali, G.: Atmospheric fate of $\mathrm{OH}$ initiated oxidation of terpenes, Reaction mechanism of a-pinene degradation and secondary organic aerosol formation, J. Environ. Manage., 75, 275-282, 2005.

May, K. R.: The collison nebulizer: Description, performance and application, J. Aerosol Sci., 4, 235-243, 1973.

Noyes, W. A.: n-butyl nitrite, Org. Synth., 16, 7-8, 1936.

Pöschl, U.: Atmospheric aerosols: Composition, transformation, climate and health effects, Angewandte Chemie-International Edition, 44, 7520-7540, 2005.

Pratt, K. A., Mayer, J. E., Holecek, J. C., Moffet, R. C., Sanchez, R. O., Rebotier, T. P., Furutani, H., Gonin, M., Fuhrer, K., Su, Y., Guazzotti, S., and Prather, K. A.: Development and characterization of an aircraft aerosol time-of-flight mass spectrometer, Anal. Chem., 81, 1792-1800, 2009.

Reinnig, M., Warnke, J., and Hoffmann, T.: Identification of organic hydroperoxides and hydroperoxy acids in secondary organic aerosol formed during the ozonolysis of different monoterpenes and sesquiterpenes by on-line analysis using atmospheric pressure chemical ionization ion trap mass spectrometry, Rapid Commun. Mass Sp., 23, 1735-1741, 2009.

Ruiz, P. A., Lawrence, J. E., Ferguson, S. T., Wolfson, J. M., and Koutrakis, P.: A counter-current parallel-plate membrane denuder for the non-specific removal of trace gases, Environ. Sci. Technol., 40, 5058-5063, 2006.

Soderholm, S. C.: Analysis of diffusion battery data, J. Aerosol Sci., 10, 163-175, 1979.

Tanimoto, H., Aoki, N., Inomata, S., Hirokawa, J., and Sadanaga, Y.: Development of a PTR-TOFMS instrument for real-time measurements of volatile organic compounds in air, Int. J. Mass Spectrom., 263, 1-11, 2007.

Temime, B., Healy, R. M., and Wenger, J. C.: A denuder-filter sampling technique for the detection of gas and particle phase carhonyl compounds, Environ. Sci. Technol., 41, 6514-6520, 2007.

Thomson, D. S., Schein, M. E., and Murphy, D. M.: Particle analysis by laser mass spectrometry WB-57F instrument overview, Aerosol Sci. Tech., 33, 153-169, 2000.

Warscheid, B., Kuckelmann, U., and Hoffmann, T.: Direct quantitative analysis of organic compounds in the gas and particle phase using a modified atmospheric pressure chemical ionization source in combination with ion trap mass spectrometry, Anal. Chem., 75, 1410-1417, 2003.

Woods, E., Smith, G. D., Dessiaterik, Y., Baer, T., and Miller, R. E.: Quantitative detection of aromatic compounds in single aerosol particle mass spectrometry, Anal. Chem., 73, 2317-2322, 2001.

Zhang, Y.: Indoor Air Quality Engineering, CRC Press, Boca Raton, Fla, 2005. 